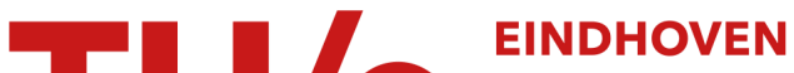 UNIVERSITY OF TECHNOLOGY
}

\section{Price of anarchy in the Markovian single server queue}

Citation for published version (APA):

Gilboa-Freedman, G., Hassin, R., \& Kerner, Y. (2008). Price of anarchy in the Markovian single server queue. (Report Eurandom; Vol. 2008043). Eurandom.

Document status and date:

Published: 01/01/2008

\section{Document Version:}

Publisher's PDF, also known as Version of Record (includes final page, issue and volume numbers)

\section{Please check the document version of this publication:}

- A submitted manuscript is the version of the article upon submission and before peer-review. There can be important differences between the submitted version and the official published version of record. People interested in the research are advised to contact the author for the final version of the publication, or visit the $\mathrm{DOI}$ to the publisher's website.

- The final author version and the galley proof are versions of the publication after peer review.

- The final published version features the final layout of the paper including the volume, issue and page numbers.

Link to publication

\section{General rights}

Copyright and moral rights for the publications made accessible in the public portal are retained by the authors and/or other copyright owners and it is a condition of accessing publications that users recognise and abide by the legal requirements associated with these rights.

- Users may download and print one copy of any publication from the public portal for the purpose of private study or research.

- You may not further distribute the material or use it for any profit-making activity or commercial gain

- You may freely distribute the URL identifying the publication in the public portal.

If the publication is distributed under the terms of Article $25 \mathrm{fa}$ of the Dutch Copyright Act, indicated by the "Taverne" license above, please follow below link for the End User Agreement:

www.tue.nl/taverne

\section{Take down policy}

If you believe that this document breaches copyright please contact us at:

openaccess@tue.nl

providing details and we will investigate your claim. 


\title{
Price of Anarchy in the Markovian Single Server Queue
}

\author{
Gail Gilboa-Freedman, ${ }^{*}$ Refael Hassin, Yoav Kerner ${ }^{\dagger}$
}

\begin{abstract}
The Price of Anarchy (PoA) is the ratio of optimal and equilibrium social welfare. We consider the most fundamental queueing model involving customers' decisions, as studied by Naor (1969). We show that the PoA has an odd behavior as a function of the system's congestion. In particular, when the ratio of the potential arriving rate and the service rate is at most $0.98, \operatorname{PoA}<1.5$. As the arrival rate comes close to the service rate, the bound on the PoA increases to 2 , and when the ratio of the potential arriving rate and the service rate exceeds $1, \mathrm{PoA}$ is unbounded.
\end{abstract}

\section{Introduction}

Congested systems in general, and queueing systems in particular, may involve decision making by individuals that affect not only the welfare of those who act but also the welfare of other individuals in the system. Individuals are motivated then to act strategically in order to maximize their own welfare given the strategies adopted by others. The most common solution concept in such models is the Nash equilibrium. The equilibrium solution is often different from the solution which maximizes overall social welfare. A common measure to this inefficiency is the Price of Anarchy (PoA), i.e., the ratio between the social welfare under optimum and the social welfare under equilibrium ${ }^{1}$. The PoA has been studied in many settings, see for example $[1,7,9]$.

This paper is the first to investigate the PoA in the most fundamental queueing model that involves customers' decision making. The equilibrium and socially optimal behavior in this model were analyzed in the seminal paper of Naor [6]. The model assumes a First-Come First-Served queue,

${ }^{*}$ Department of Statistics and Operation Research, Tel Aviv University, Israel. Emails: gailgf@post.tau.ac.il, hassin@post.tau.ac.il

${ }^{\dagger}$ EURANDOM, Eindhoven University of Technology, The Netherlands. Email:kerner@eurandom.tue.nl.

${ }^{1}$ When the equilibrium is not unique, the convention is inserting the worse equilibrium, from the society's point of view. 
observable by the decision maker, with a single server, Poisson arrivals, exponential service times, linear waiting costs and fixed rewards from obtaining service. Balking is associated with zero reward. The equilibrium solution in this model is very simple since there exists a dominant pure threshold strategy. Namely, for some integer $n$, an arriving customer joins the queue if and only if the observed queue length upon arrival is shorter than $n$, and this strategy maximizes the individual's expected welfare no matter what strategies are adopted by the others. The optimal behavior is also characterized by a threshold strategy. Moreover Naor observed that its threshold parameter is in general smaller than that of the equilibrium strategy. This result is robust in the sense that it also holds for more general queuing models , [4], [5] and [8].

Following Naor's notation (see also [2]), we denote the arrival rate by $\lambda$, and the service rate by $\mu$. There is a reward of value $R$ the customer obtains upon completing service, and a cost of $C$ per unit of time spent waiting or in service. The system's utilization factor is $\rho=\frac{\lambda}{\mu}$, and we use $\nu_{s}=\frac{R \mu}{C}$ to denote the value of service in terms of expected waiting cost during a service duration. ${ }^{2}$

The model's parameters can be normalized so that the only relevant parameters are $\rho$ and $\nu_{s}$. Note that the model doesn't need to assume $\rho<1$ for stability, and in fact this number is of no significance in Naor's results. It comes therefore as a surprise that if we impose no restrictions on $\nu_{s}$ then PoA is bounded if and only if $\rho \leq 1$. The behavior of PoA as a function of the utilization factor $\rho$ can be roughly separated into three regions. As mentioned, for $\rho>1 \mathrm{PoA}$ is unbounded. We derive the exact value of PoA for $\rho<0.9818$, and show that everywhere in this range $\mathrm{PoA}<1.48635$. The most interesting behavior of PoA is observed in the range $0.9818<\rho<1$, where it sharply increases but remains bounded by 2 .

One can summarize therefore, that since in most real situations $\rho<0.98$, the price of anarchy in the single server queue is relatively small, compared with other models discussed in the literature.

Finally, we mention that the only paper that deals with PoA in a queuing system is a study by Haviv and Roughgarden [3], who consider a multiserver queueing system with exponential service times, and show that the PoA is bounded from above by the number of servers used under social optimization.

\section{PoA general behavior}

The equilibrium and optimal thresholds, $n_{e}$ and $n^{*}$ respectively, and their associated social welfare, have been studied by Naor [6]. It is straightforward

\footnotetext{
${ }^{2}$ To avoid triviality, $\nu_{s} \geq 1$. Otherwise, an arriving customer would balk even if the system is empty.
} 
that

$$
n_{e}=\left\lfloor\frac{R \mu}{C}\right\rfloor=\left\lfloor\nu_{s}\right\rfloor
$$

Define

$$
g(\nu)=\frac{\nu(1-\rho)-\rho\left(1-\rho^{\nu}\right)}{(1-\rho)^{2}}
$$

then $n^{*}=\left\lfloor\nu^{*}\right\rfloor$, where $\nu^{*}$ is the unique solution to

$$
g(\nu)=\nu_{s} .
$$

Naor showed that $n^{*} \leq n_{e}$. Moreover, $n^{*}=n_{e}$ if and only if $n_{e}=1$.

The social welfare associated with a threshold $n$ is

$$
S_{n}=R \lambda \frac{1-\rho^{n}}{1-\rho^{n+1}}-C\left[\frac{\rho}{1-\rho}-\frac{(n+1) \rho^{n+1}}{1-\rho^{n+1}}\right] .
$$

PoA is defined as the ratio of $S_{n^{*}}$ and $S_{n_{e}}$, i.e.,

$$
\begin{aligned}
\operatorname{PoA}\left(\rho, \nu_{s}\right)= & \frac{\frac{1-\rho^{n^{*}}}{1-\rho^{n^{*}+1}}-\frac{1}{\nu_{s}}\left[\frac{1}{1-\rho}-\frac{\left(n^{*}+1\right) \rho^{n^{*}}}{1-\rho^{n^{*}+1}}\right]}{\frac{1-\rho^{\left\lfloor\nu_{s}\right\rfloor}}{1-\rho^{\left\lfloor\nu_{s}\right\rfloor+1}}-\frac{1}{\nu_{s}}\left[\frac{1}{1-\rho}-\frac{\left(\left\lfloor\nu_{s}\right\rfloor+1\right) \rho^{\left\lfloor\nu_{s}\right\rfloor}}{1-\rho^{\left\lfloor\nu_{s}\right\rfloor+1}}\right]} \\
& =\frac{p_{\text {join }}\left(n^{*}\right)-\frac{1}{\nu_{s}} q\left(n^{*}\right)}{p_{\text {join }}\left(n_{e}\right)-\frac{1}{\nu_{s}} q\left(n_{e}\right)}
\end{aligned}
$$

where $q(n)$ is the expected queue length under a threshold $n$, and $p_{\text {join }}(n)$ is the probability that an arriving customer joins the queue.

$\operatorname{PoA}\left(\rho, \nu_{s}\right)$ is shown in Figures 1 and 2. In the following subsections, we take a closer look at these graphs. 


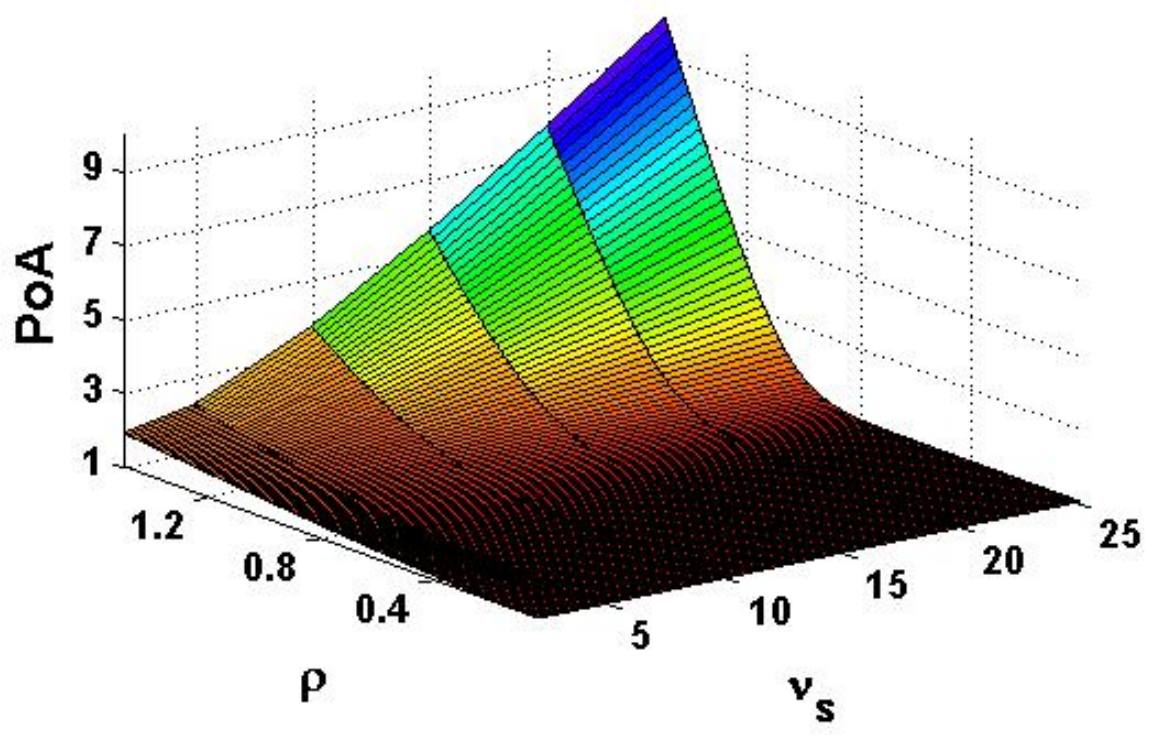

Figure 1: PoA Vs. $\rho$ and $\nu_{s}$

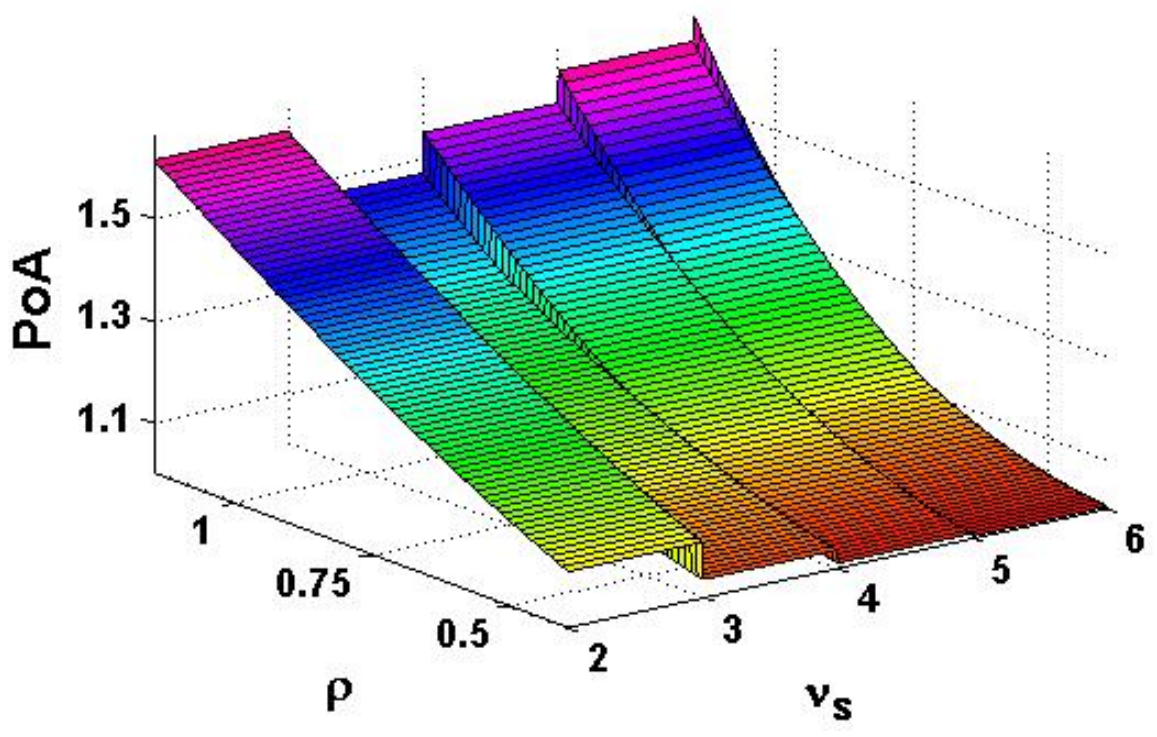

Figure 2: PoA Vs. $\rho$ and $\nu_{s}$ - zoom in 


\section{PoA as a function of $\nu_{s}$}

For $\rho<1$, we observe two local maxima, one of them when $\nu_{s}=2$. It is demonstrated in Figure 3 for three different values of $\rho$. In the next section we'll show that, except for a small range of $\rho$ values near but below 1 , the global maximum is achieved when $\nu_{s}=2$.

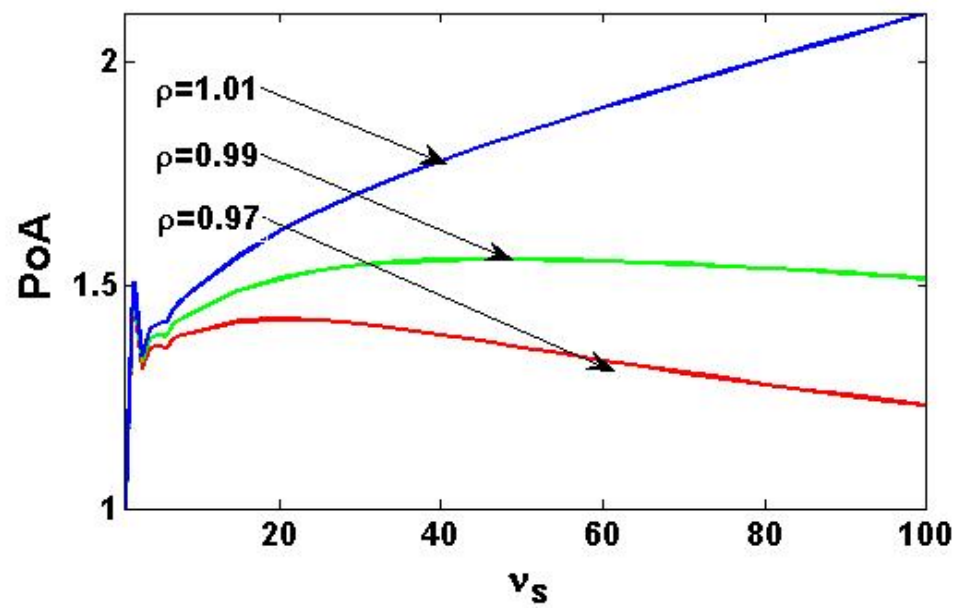

Figure 3: $\operatorname{PoA}\left(\nu_{s}\right)$ for various values of $\rho$

For $\rho \geq 1$ the PoA is monotone increasing when $\nu_{s} \rightarrow \infty$. The asymptotic behavior is described by the following lemmas:

Lemma 3.1. $\lim _{\nu_{s} \rightarrow \infty} \operatorname{PoA}\left(1, \nu_{s}\right)=2$.

Proof. For $\rho=1$, the queue length distribution is uniform on $0,1, \ldots, n$ and in particular, $q(n)=n / 2$ and $p_{\text {join }}=n /(n+1)$.

Consider the function

$$
h(x)=\frac{x}{x+1}-\frac{x}{2 \nu_{s}} .
$$

A continuous (with respect to $x$ ) analysis of this expression yields that it is maximized by $x=\sqrt{2 \nu_{s}}-1$. Thus, $S_{n *}=h(n *) \leq h\left(\sqrt{2 \nu_{s}}-1\right)$.

Also, by $n^{*}<\nu_{s}$ and concavity of $h(x), S_{n_{e}}=h\left(\left\lfloor\nu_{s}\right\rfloor\right) \geq h\left(\nu_{s}\right)$.

Therefore,

$$
\operatorname{PoA}\left(1, \nu_{s}\right) \leq \frac{\frac{\sqrt{2 \nu_{s}}-1}{\sqrt{2 \nu_{s}}}-\frac{\sqrt{2 \nu_{s}}-1}{2 \nu_{s}}}{\frac{\nu_{s}}{\nu_{s}+1}-\frac{1}{2}},
$$

which tends to 2 when $\nu_{s} \rightarrow \infty$.

Lemma 3.2. $\forall \rho>1, \operatorname{PoA}\left(\rho, \nu_{s}\right) \rightarrow \infty$ for $\nu_{s} \rightarrow \infty$. 
Proof. When $\rho>1$ and when $\nu_{s} \rightarrow \infty$, by (1)

$$
n^{*}=\log _{\rho} \nu_{s}+o\left(\log _{\rho} \nu_{s}\right)
$$

By $(2)$

$$
\operatorname{PoA}\left(\rho, \nu_{s}\right) \sim \frac{\frac{1-\rho^{\log _{\rho} \nu_{s}}}{1-\rho^{\log _{\rho} \nu_{s}+1}}-\frac{1}{\nu_{s}}\left[\frac{1}{1-\rho}-\frac{\left(\log _{\rho} \nu_{s}+1\right) \rho^{\log _{\rho} \nu_{s}}}{1-\rho^{\log _{\rho} \nu_{s}+1}}\right]}{\frac{\left.1-\rho \nu_{s}\right\rfloor}{1-\rho^{\left\lfloor\nu_{s}\right\rfloor+1}}-\frac{1}{\nu_{s}}\left[\frac{1}{1-\rho}-\frac{\left(\left\lfloor\nu_{s}\right\rfloor+1\right) \rho \nu^{\left\lfloor\nu_{s}\right\rfloor}}{1-\rho^{\left\lfloor\nu_{s}\right\rfloor+1}}\right]} .
$$

Since

$$
\begin{gathered}
\frac{1-\rho^{\log _{\rho} \nu_{s}}}{1-\rho^{\log _{\rho} \nu_{s}+1}}-\frac{1}{\nu_{s}}\left[\frac{1}{1-\rho}-\frac{\left(\log _{\rho} \nu_{s}+1\right) \rho^{\log _{\rho} \nu_{s}}}{1-\rho^{\log _{\rho} \nu_{s}+1}}\right]=\frac{1-\nu_{s}}{1-\rho \nu_{s}}-\frac{1}{\nu_{s}}\left[\frac{1}{1-\rho}-\frac{\left(\log _{\rho} \nu_{s}+1\right) \nu_{s}}{1-\rho \nu_{s}}\right] \sim \\
\frac{1}{\rho}-\frac{1}{\nu_{s}}\left[\frac{1}{1-\rho}+\frac{\log _{\rho} \nu_{s}}{\rho}\right]=\frac{\nu_{s}(1-\rho)-\rho-(1-\rho) \log _{\rho} \nu_{s}}{\nu_{s}(1-\rho) \rho} \sim \frac{1}{\rho}
\end{gathered}
$$

and

$$
\begin{aligned}
& \frac{1-\rho^{\left\lfloor\nu_{s}\right\rfloor}}{1-\rho^{\left\lfloor\nu_{s}\right\rfloor+1}}-\frac{1}{\nu_{s}}\left[\frac{1}{1-\rho}-\frac{\left(\left\lfloor\nu_{s}\right\rfloor+1\right) \rho\left\lfloor\nu_{s}\right\rfloor}{1-\rho\left\lfloor\nu_{s}\right\rfloor+1}\right] \sim \frac{1}{\rho}-\frac{1}{\nu_{s}}\left[\frac{1}{1-\rho}+\frac{\left\lfloor\nu_{s}\right\rfloor}{\rho}\right] \\
= & \frac{\nu_{s}(1-\rho)-\rho-(1-\rho)\left\lfloor\nu_{s}\right\rfloor}{\nu_{s}(1-\rho) \rho}=\frac{\left(\nu_{s}-\left\lfloor\nu_{s}\right\rfloor\right)(1-\rho)-\rho}{\nu_{s}(1-\rho) \rho}<\frac{1-2 \rho}{\nu_{s}(1-\rho) \rho} \underset{\nu_{s} \rightarrow \infty}{\longrightarrow} 0,
\end{aligned}
$$

we conclude that

$$
\operatorname{PoA}\left(\rho, \nu_{s}\right) \underset{\nu_{s} \rightarrow \infty}{\longrightarrow} \infty
$$

In contrast, $\forall \rho<1$, the $\mathrm{PoA}$ is monotone decreasing to 1 when $\nu_{s} \rightarrow \infty$.

Lemma 3.3. $\forall \rho<1, \lim _{\nu_{s} \rightarrow \infty} \operatorname{PoA}\left(\rho, \nu_{s}\right)=1$.

Proof. When $\rho \leq 1$, the solution of Equation (1) satisfies

$$
\lim _{\nu_{s} \rightarrow \infty} \frac{n^{*}}{\nu_{s}}=1-\rho .
$$

Substituting this relation into (2) gives:

$$
\begin{aligned}
\lim _{\nu_{s} \rightarrow \infty} & \operatorname{PoA}\left(\rho, \nu_{s}\right)= \\
& \frac{\frac{1-\rho^{\nu_{s}}(1-\rho)}{1-\rho^{\nu_{s}(1-\rho)+1}}-\frac{1}{r}\left[\frac{1}{1-\rho}-\frac{\left(\nu_{s}(1-\rho)+1\right) \rho^{\nu_{s}(1-\rho)}}{1-\rho^{\nu_{s}}(1-\rho)+1}\right]}{\frac{1-\rho^{\left\lfloor\nu_{s}\right\rfloor}}{1-\rho^{\left\lfloor\nu_{s}\right\rfloor+1}}-\frac{1}{\nu_{s}}\left[\frac{1}{1-\rho}-\frac{\left(\left\lfloor\nu_{s}\right\rfloor+1\right) \rho^{\left.\nu_{s}\right\rfloor}}{1-\rho^{\left\lfloor\nu_{s}\right\rfloor+1}}\right]} \underset{\nu_{s} \rightarrow \infty}{\longrightarrow} 1
\end{aligned}
$$




\section{PoA as a function of $\rho$}

In this section we consider the upper envelope of the function $\operatorname{PoA}\left(\rho, \nu_{s}\right)$ for $\nu_{s} \geq 1$ :

$$
\operatorname{PoA}(\rho)=\sup _{\nu_{s} \geq 1} \operatorname{PoA}\left(\rho, \nu_{s}\right) .
$$

Lemma 4.1. For any $\rho>1, \operatorname{PoA}(\rho)$ is unbounded.

Proof. By Lemma 3.2.

Remark. Under the limit case, when $\rho \rightarrow \infty$, given any threshold $n$, the number of customers in the system is always $n$. This is because at any departure instant, an arrival occurs, due to the infinite arrival rate. The social welfare per unit of time is then $R \mu-n C$. Thus, $n^{*}=1$. An explanation to the latter is that in general, under optimization, customers are allowed to wait to prevent idleness of the server in the future. When the arrival rate is infinite, idleness never occurs. On the other hand, for fixed $\mu, C$ and $R, n_{e}$ does not depend on $\lambda$ and $n_{e}$ remains the same. The PoA is

$$
\frac{R \mu-C}{R \mu-C n_{e}}=\frac{1-\frac{1}{\nu_{s}}}{1-\frac{\left\lfloor\nu_{s}\right\rfloor}{\nu_{s}}}=\frac{\nu_{s}-1}{\nu_{s}-\left\lfloor\nu_{s}\right\rfloor} \stackrel{\nu_{s} \rightarrow \infty}{\longrightarrow} \infty
$$

Lemma 4.2. $\operatorname{PoA}(1)=2$.

Proof. By (4)

$$
P o A\left(1, \nu_{s}\right) \leq \frac{\frac{\sqrt{2 \nu_{s}}-1}{\sqrt{2 \nu_{s}}}-\frac{\sqrt{2 \nu_{s}}-1}{2 \nu_{s}}}{\frac{\nu_{s}}{\nu_{s}+1}-\frac{1}{2}} \leq \frac{\left(\sqrt{2 \nu_{s}}-1\right)^{2}\left(\nu_{s}+1\right)}{\nu_{s}\left(\nu_{s}-1\right)} .
$$

For $\nu_{s}=1,2,3$, the righthand side is less than 1.5, and for $\nu_{s}>2+\sqrt{3}$ its derivative is positive. Therefore, $\operatorname{Po} A\left(1, \nu_{s}\right)$ is bounded by the limit when $\nu_{s}$ goes to infinity, which is 2 .

The following Lemmas will be used in the proof of Theorem 4.5.

Lemma 4.3. For any $\rho<1$, if $\operatorname{PoA}\left(\rho, \nu_{s}\right)$ is maximized at $\nu_{s}$ then $\nu_{s}$ is an integer.

Proof. We show that PoA is strictly monotone decreasing with $\nu_{s}$ in the range where $n_{e}$ is fixed, i.e. $\nu_{s} \in[n, n+1)$. This range is divided to a finite number of intervals, such that in each interval $n^{*}$ is also fixed. The PoA is continuous where $n^{*}$ changes because at these values $S_{n *}=S_{n *+1}$. Therefore it is sufficient to show that PoA is strictly monotone decreasing with $\nu_{s}$ in each of the intervals, where both $n^{*}$ and $n_{e}$ are fixed. 
Consider two values $\nu_{s}^{1}<\nu_{s}^{2}$ in such interval. The derivative of (3) with respect to $\nu_{s}$ is

$$
\operatorname{Po}^{\prime}\left(\rho, \nu_{s}\right)=\frac{q\left(n^{*}\right) p_{\text {join }}\left(n_{e}\right)-q\left(n_{e}\right) p_{\text {join }}\left(n^{*}\right)}{\nu_{s}^{2}\left[p_{\text {join }}\left(n_{e}\right)-\frac{1}{\nu_{s}} q\left(n_{e}\right)\right]^{2}}
$$

which is negative when

$$
q\left(n^{*}\right) p_{\text {join }}\left(n_{e}\right)<q\left(n_{e}\right) p_{\text {join }}\left(n^{*}\right)
$$

or

$$
\left(\frac{1}{1-\rho}-\frac{\left(n^{*}+1\right) \rho^{n^{*}}}{1-\rho^{n^{*}+1}}\right)\left(\frac{1-\rho^{n_{e}}}{1-\rho^{n_{e}+1}}\right)<\left(\frac{1}{1-\rho}-\frac{\left(n_{e}+1\right) \rho^{n_{e}}}{1-\rho^{n_{e}+1}}\right)\left(\frac{1-\rho^{n^{*}}}{1-\rho^{n^{*}+1}}\right)
$$

This inequality holds when

$$
\begin{aligned}
& \left(1-\rho^{n_{e}}\right)\left(1-\rho^{n^{*}+1}\right)-\left(n^{*}+1\right) \rho^{n^{*}}\left(1-\rho^{n_{e}}\right)(1-\rho)- \\
& \left(1-\rho^{n^{*}}\right)\left(1-\rho^{n_{e}+1}\right)+\left(n_{e}+1\right) \rho_{e}^{n}\left(1-\rho^{n^{*}}\right)(1-\rho)<0
\end{aligned}
$$

Simplifying the last expression, it is left to show that

$$
n_{e} \rho^{n_{e}}\left(1-\rho^{n^{*}}\right)-n^{*} \rho^{n^{*}}\left(1-\rho_{e}^{n}\right)<0
$$

or

$$
\frac{\rho^{-n^{*}}\left(1-\rho^{n^{*}}\right)}{n^{*}}-\frac{\rho^{-n_{e}}\left(1-\rho^{n_{e}}\right)}{n_{e}}<0
$$

which is true since $n^{*}<n_{e}$ and $\frac{\rho^{-n}\left(1-\rho^{n}\right)}{n}$ is increasing with $n$ when $\rho<1$. We conclude that $\operatorname{PoA}\left(\rho, \nu_{s}\right)$ is strictly decreasing with $\nu_{s} \in[n, n+1]$. In particular this means that the maximum value of PoA in this range is obtained at $\nu_{s}=n$.

Lemma 4.4. For $\rho<1, \frac{1+(n+1) \rho^{n}-\rho^{n+1}(n+2)}{1-\rho^{n+1}}$ is a monotone decreasing function of $n$.

Proof. Note that

$$
\frac{1+(n+1) \rho^{n}-\rho^{n+1}(n+2)}{1-\rho^{n+1}}=1+\frac{(n+1)(1-\rho) \rho^{n}}{1-\rho^{n+1}}=1+\frac{n+1}{\sum_{i=0}^{n} \rho^{i-n}}=1+\frac{n+1}{\sum_{i=0}^{n} \rho^{-i}} .
$$

Since $\rho<1, \frac{\sum_{i=0}^{n} \rho^{-i}}{n+1}$ is an average of an increasing sequence, and hence it is increasing with $n$. Thus, its inverse is decreasing with $n$. 
Theorem 4.5. $\forall \rho<1, \operatorname{PoA}(\rho)<2$.

Proof. By (2), we need to prove that $\forall \rho<1$ and $r \geq 1$ :

$$
\begin{array}{r}
\frac{1-\rho^{n^{*}}}{1-\rho^{n^{*}+1}}-\frac{1}{\nu_{s}}\left(\frac{1}{1-\rho}-\left(n^{*}+1\right) \frac{\rho^{n^{*}}}{1-\rho^{n^{*}+1}}\right) \leq \\
2\left[\frac{1-\rho^{\left\lfloor\nu_{s}\right\rfloor}}{1-\rho^{\left\lfloor\nu_{s}\right\rfloor+1}}-\frac{1}{\nu_{s}}\left(\frac{1}{1-\rho}-\left(\left\lfloor\nu_{s}\right\rfloor+1\right) \frac{\rho^{\left\lfloor\nu_{s}\right\rfloor}}{1-\rho^{\left\lfloor\nu_{s}\right\rfloor+1}}\right)\right] .
\end{array}
$$

By Lemma 4.3 it is sufficient to consider $\nu_{s}=\left\lfloor\nu_{s}\right\rfloor$. Since $\nu_{s}$ is an integer and $n^{*}<\nu_{s}$, it would be sufficient to prove that for any two integers $n<m$ :

$$
\begin{array}{r}
\frac{1-\rho^{n}}{1-\rho^{n+1}}-\frac{1}{m}\left(\frac{1}{1-\rho}-(n+1) \frac{\rho^{n}}{1-\rho^{n+1}}\right) \leq \\
2\left[\frac{1-\rho^{m}}{1-\rho^{m+1}}-\frac{1}{m}\left(\frac{1}{1-\rho}-(m+1) \frac{\rho^{m}}{1-\rho^{m+1}}\right)\right] .
\end{array}
$$

Since $\frac{1-\rho^{n}}{1-\rho^{n+1}}$ is monotone increasing in $n$, it is sufficient to prove

$$
\frac{1}{m} \frac{1}{1-\rho}+\frac{1}{m}(n+1) \frac{\rho^{n}}{1-\rho^{n+1}} \leq \frac{1-\rho^{m}}{1-\rho^{m+1}}+\frac{2}{m}(m+1) \frac{\rho^{m}}{1-\rho^{m+1}} .
$$

Multiplying by $m(1-\rho)$, it is sufficient to prove

$$
\frac{1+(n+1) \rho^{n}-\rho^{n+1}(n+2)}{1-\rho^{n+1}} \leq \frac{m+\rho^{m}(m+2)}{1-\rho^{m+1}}(1-\rho) .
$$

By Lemma 4.4, the left-hand side is monotone decreasing with $n$. Therefore it is sufficient to show that the inequality holds for $n=1$ :

$$
\frac{1+2 \rho-3 \rho^{2}}{1-\rho^{2}}<\frac{m+\rho^{m}(m+2)(1-\rho)}{1-\rho^{m+1}}
$$

or

$$
0<2+(m-3)(1+\rho)+(m+1) \rho^{m}+\rho^{m}(1+\rho)-(m-1) \rho^{m+2},
$$

which is true by

$$
(m+1) \rho^{m}>(m-1) \rho^{m+2} .
$$

The behavior of $\operatorname{PoA}(\rho)$ in the range $\rho<1$ is as shown in Figure 4. One sees that except for a small range near $\rho=1, \operatorname{PoA}(\rho)$ is bounded by 1.5 . This bound is smaller than the bound in Theorem 4.5. However, when $\rho$ becomes close to 1 the bound sharply increases to 2 . To understand this oddness, we examine the behavior of the PoA for various values of $\nu_{s}$.

Figure 5 demonstrates that when $\rho<0.98175, \operatorname{PoA}(\rho)=\operatorname{PoA}(\rho, 2)$. In contrast, when $\rho \in[0.98175,1], \operatorname{PoA}(\rho)$ is not equal to a single function $\operatorname{PoA}\left(\rho, \nu_{s}\right)$. Instead, there is an infinite number of functions which define the upper envelope of the PoA in this range. 


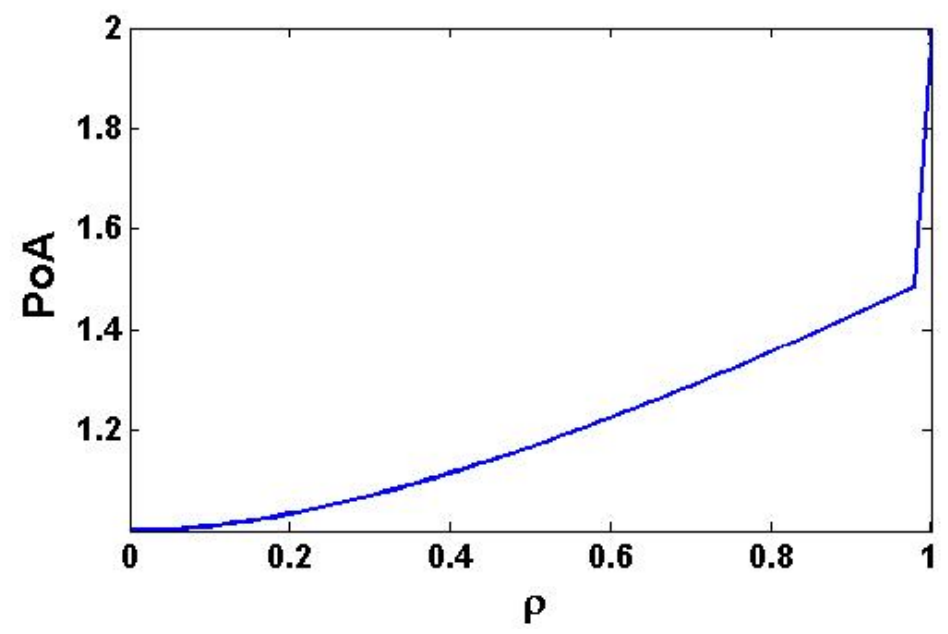

Figure 4: PoA Vs. $\rho$

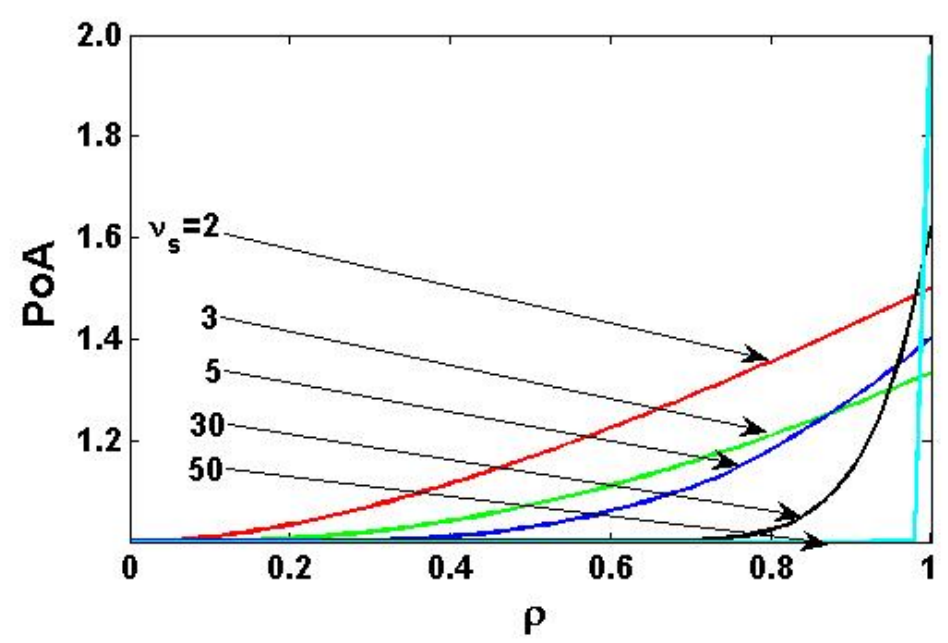

Figure 5: $\operatorname{PoA}(\rho)$ for various values of $\nu_{s}$

Observation 4.6. If $\rho \in[0,0.98175], \operatorname{PoA}\left(\rho, \nu_{s}\right) \leq \frac{1+\rho+\rho^{2}}{1+\rho}<1.48635$

Proof. We observe that $\operatorname{PoA}(\rho)=\operatorname{PoA}(\rho, 2)$ when $\rho<0.98175$. Substituting $\nu_{s}=2$ in $(2)$ we have

$\operatorname{PoA}\left(\rho, \nu_{s}\right) \leq \frac{1+\rho+\rho^{2}}{1+\rho}$. We also have that $\mathrm{PoA}$ is uniformly bounded by the maximum of $\frac{1+\rho+\rho^{2}}{1+\rho}$ over $[0,0.98175]$ which is 1.48635 . 


\section{References}

[1] M.M. Halldorsson, J.Y. Halpern, L.E. Li and V.S. Mirrokni, "On spectrum sharing games," Proceedings of the Twenty-Third Annual ACM Symposium on Principles of Distributed Computing 107-114 (2004).

[2] R. Hassin and M. Haviv, To Queue or not to Queue: Equilibrium Behavior in Queueing Systems, Kluwer International Series, 2003.

[3] M. Haviv and T. Roughgarden, "The price of anarchy in an exponential multi-server," Operations Research Letters 35 421-426, (2007).

[4] N.C. Knudsen, "Bounding the inefficiency of equilibria in nonatomic congestion games," Econometrica 47 515-528, (1972).

[5] S. A. Lippman and S. Stidham, "Individual versus social optimization in exponential congested systems," Operations Research 25 233-247, (1977).

[6] P. Naor, "The regulation of queue size by levying tolls," Econometrica 37 15-24, (1969).

[7] T. Roughgarden and E. Tardos, "Individual and social optimization in a multiserver queue with a general cost-benefit structure," Games and Economic Behavior 47 389-403, (2004).

[8] S. Stidham, "Socially and individually optimal control of arrivals to a GI/M/1 queue," Management Science 47 1598-1610, (1978).

[9] A. Vetta, "Nash equilibria in competitive societies, with applications to facility location, traffic routing and auctions," Proceedings of the 43rd Annual IEEE Symposium on Foundations of Computer Science, 416-425, (2002). 